\title{
Computed Tomography Arteriography for Detecting the Origin of the Inferior Pyloric Artery in Patients with Gastric Cancer
}

\author{
Zhi-Long Wang, MD ${ }^{1 *}$, Ru-Lin Miao, MD ${ }^{2 *}$, Chao Gao, MD², Lei Tang, MD ${ }^{1}$, Zi-Yu Li, MD², \\ Ying-Shi Sun, MD ${ }^{1}$, Jia-Fu Ji, MD, FACS ${ }^{2}$ \\ Departments of ${ }^{1}$ Radiology and ${ }^{2}$ Gastrointestinal Surgery, Peking University Cancer Hospital \& Institute, Beijing, China; Key Laboratory of \\ Carcinogenesis and Translational Research (Ministry of Education), Beijing, China
}

Objective: To analyze the detection rate of the inferior pyloric artery (IPA) in patients with gastric cancer by computed tomography arteriography (CTA).

Materials and Methods: Fifty-four patients (48 males and 6 females; mean age, $59.0 \pm 1.5$ years) who had undergone radical gastrectomy for gastric cancer from September 2016 to July 2017 at our institution were recruited prospectively. Patients underwent abdominal contrast-enhanced CT scans and CTA imaging reconstruction before the operation. The origin of the IPA in all cases was determined by a radiologist based on CTA images and verified by the surgeon. The accuracy of CTA in diagnosing the origin of the IPA was calculated. Dominant vessels of the origin were analyzed.

Results: IPAs were detected by CTA in 51 patients (94.4\%). Among these, IPAs originated from the right gastroepiploic artery (RGEA) (24 cases), the gastroduodenal artery (GDA) (4 cases), and the anterior superior pancreaticoduodenal artery (ASPDA) (20 cases). In the remaining 3 cases, the IPAs contained two branches originating from the RGEA and ASPDA, respectively. During surgery, in $2(3.7 \%)$ of the 54 cases of gastric cancer, IPAs could not be detected; the IPAs originated from the RGEA (22 cases), GDA (5 cases), and ASPDA (24 cases). One case had an IPA originating from both the RGEA and the GDA. Finally, the accuracy of CTA in diagnosing the origin artery of the IPA was $85.2 \%(46 / 54)$.

Conclusion: CTA can detect the origin of the IPA accurately, which can aid surgeons while performing pylorus-preserving operations.

Keywords: Gastric cancer; Surgical anatomy; Inferior pyloric artery; Computed tomography

\section{INTRODUCTION}

As the rate of lymph node metastasis of early gastric cancer is relatively low and the survival is good after surgical treatment, the extent of lymph node dissection and gastric resection could be reduced to preserve the digestive function of the stomach. Recently, some groups indicated that pylorus-preserving gastrectomy (PPG) could be carried out for early gastric cancer if the blood flow of the pylorus can be preserved. The location of these tumors should limit to the middle third of the stomach. By preserving pyloric functions, patients who undergo PPG have a lower incidence of postgastrectomy disorders, including dumping syndrome, alkaline reflux, disturbed bowel habits, and frequent flatus,

Received April 26, 2018; accepted after revision June 13, 2018.

This study was supported by the Project of Gastric Cancer Research, Funding of Beijing Municipal Science \& Technology Commission (D171100006517004); Beijing Municipal science \& Technology Commission (Z161100000516060, Z151100004015070); Beijing Municipal Administration of Hospital's Youth Program (QML20161102); Beijing Organization Department's talents project (2017000021469G279). *These authors contributed equally to this work.

Corresponding author: Jia-Fu Ji, MD, FACS, Department of Gastrointestinal Surgery, Peking University Cancer Hospital \& Institute. No.52, Fucheng Road, Haidian District, Beijing 100142, China.

- Tel: (8610) 88196606• Fax: (8610) 88196606• E-mail: jijiafu@hsc.pku.edu.cn

This is an Open Access article distributed under the terms of the Creative Commons Attribution Non-Commercial License (https:// creativecommons.org/licenses/by-nc/4.0) which permits unrestricted non-commercial use, distribution, and reproduction in any medium, provided the original work is properly cited. 
than patients who undergo conventional distal gastrectomy.

In this operation, the preservation of the inferior pyloric artery (IPA) is a crucial technique to ensure the blood supply of the pylorus (1-3). Detecting the IPA and identifying its origin and route by examination before surgery may be useful for the surgeon while performing successful PPG. Some reports indicated that computed tomography arteriography (CTA) could detect the main perigastric arteries including the left gastric artery, right gastric artery, left gastroepiploic artery, and right gastroepiploic artery (RGEA), thereby providing adequate guidance for the operation (4-6). The reconstruction of the $\mathrm{CT}$ angiogram has been effective in assessing the perigastric vascular anatomy before laparoscopy-assisted gastrectomy for gastric cancer. However, no report described the ability of CTA to detect the IPA, owing to its small size and since it has not been under intense focus until recently. Therefore, here, we used CTA to investigate the detection rate, origin, and variation of the IPA in patients with gastric cancer. Furthermore, we compared CTA with conventional surgery to explore its application in detecting the IPA.

\section{MATERIALS AND METHODS}

This study is a single-center data analysis based on the "IPA-Origin" trial (NCT03071237) registered on clinicaltrials.gov. This prospective study was approved by our Institutional Review Board, and all patients gave written informed consent.

\section{Patients}

Between September 2016 and July 2017, patients confirmed with gastric cancer by endoscopic biopsy and preparing to undergo radical gastrectomy were enrolled in the present study. These patients underwent contrastenhanced 64-channel CT examinations and CT arteriography 2 weeks before surgery. Patients were excluded from the study if 1) they were unable to complete the enhanced CT because of iodine allergy or 2) could not undergo surgery due to their poor physical condition.

Finally, 54 patients (48 males and 6 females; mean age \pm standard deviation, $59.0 \pm 1.5$ years) were included in this study. They consisted of 52 cases of adenocarcinoma and 2 cases of signet ring cell carcinoma.

\section{CT Protocol}

CT was performed using a 64-detector row CT scanner
(LightSpeed 64; GE Healthcare, Milwaukee, WI, USA). Each patient had been fasting for $>8$ hours prior to the $\mathrm{CT}$ examination. To enable gastric distention and reduce gastric motility, the patients were administered $8 \mathrm{~g}$ gas-producing crystals orally and an intramuscular injection of $10 \mathrm{mg}$ anisodamine (Raceanisodamine Hydrochloride Injection; Hangzhou Minsheng Pharmaceutical Co. Ltd., Hangzhou, China) 10-15 minutes before the examination. The upper abdominal unenhanced CT scans from the diaphragmatic domes to $2 \mathrm{~cm}$ below the lower margin of the air-distended gastric body were acquired with collimation of $0.625 \mathrm{~mm}$, $120 \mathrm{kVp}$, and automatic mAs. Subsequently, a total of 100 $\mathrm{mL}$ non-ionic contrast medium (iopromide, Ultravist; 370 $\mathrm{mg} / \mathrm{mL}$; Bayer, Leverkusen, Germany) was administered intravenously with an 18-gauge angiographic catheter inserted into an antecubital vein at a rate of $4 \mathrm{~mL} / \mathrm{sec}$ by an automatic injector. Arterial phase images were obtained using the bolus tracking method. The arterial phase scanning started when Hounsfield units reached 200 in the abdominal aorta at the level of bifurcation of the celiac artery. The average scanning delay time between the start of contrast material injection and the start of arterial phase scanning was 22 secconds (range 20-25 secconds). Venous phase scanning was started 35 secconds after arterial phase scanning. The arterial phase images were used in the CTA imaging procedure. The portal venous phase images were used for the routine staging diagnosis of gastric cancer.

\section{Image Analysis}

Two radiologists (with 11 and 16 years of experience in abdominal (T) performed the image analysis together on the image workstation (AW 4.5; GE Healthcare). Axial arterial phase CT images with $0.625-\mathrm{mm}$ thickness were used for CTA. In this study, the IPA was defined as the artery distributed to the anterior and posterior wall of the gastric pylorus. As the IPA was difficult to identify on 5-mm thickness images, firstly, we used $0.625-\mathrm{mm}$ thickness images of the arterial phase to search for the arterial vessel distributing to the anterior and posterior wall of the pylorus. This vessel was defined as the IPA. Then, we followed the direction of the artery backwards, to seek its original point. Thus, the maximum intensity projection (MIP) was reconstructed for the original point of the IPA in order to reveal its anatomical relationship with the other adjacent arteries. The investigators compared the identified origin of the IPA on CTA with that identified during surgery, and the diagnostic accuracy of CTA for determining the IPA was calculated. 


\section{Surgery}

All patients in this study underwent laparoscopic $(n=37)$ or open radical resection $(n=17)$ of gastric cancer. During the operation, the surgeon carefully identified the arteries distributing to the anterior and posterior wall of the pylorus as the IPA. This method was defined as the gold standard for the origin of the IPA in the present study.

\section{RESULTS}

\section{CTA Results}

CTA could not detect the IPAs in 3 of the 54 patients, while 51 patients $(94.4 \%)$ had identifiable IPAs. In these 51 cases, the IPAs originated from the RGEA (24 cases), the gastroduodenal artery (GDA) (4 cases), and the anterior superior pancreaticoduodenal artery (ASPDA) (20 cases). In the remaining 3 cases, the IPAs contained two branches originating from the RGEA and the ASPDA, respectively (Table 1, Figs. 1-4).

\section{Surgical Results}

During the surgery, of the 54 cases of gastric cancer, IPAs could be detected in 52 cases $(96.3 \%)$. The IPAs originated from the RGEA (22 cases), GDA (5 cases), and ASPDA (24 cases). Only 1 case had an IPA originating from both the RGEA and the GDA (Table 1).

\section{Statistical Analysis}

Corresponding to the surgical results, the accuracy of CTA in diagnosing the origin artery of the IPA was $85.2 \%(46 / 54)$ (Table 1).

\section{DISCUSSION}

With the development of clinical diagnosing technology, the rate of diagnosis of early gastric cancer has increased gradually. The preservation of functions is the focus of early gastric cancer surgery. In the case of early gastric cancer in the middle of the gastric body, PPG is one of the most commonly used surgical procedures for function-preserving surgery. However, PPG is an operation method that is only applicable in a small group of patients with gastric cancer. Its effectiveness is also debatable. Recently, PPG is getting more attention from surgeons. The Japanese Gastric Cancer Association has redefined the subpyloric lymph nodes of the stomach, by dividing the subpyloric lymph nodes (Group No. 6) into three subgroups, namely No. 6a, 6i, and $6 \mathrm{v}(7)$. Whether this subclassification of the subpyloric lymph nodes contributed to the increased interest in PPG is a hot topic among surgeons and remains to be studied in the future. In order to ensure the blood supply of the pylorus, the IPA needs to be preserved. Thus, identifying the anatomical origin of the IPA is crucial for PPG.

Rossi and Cova (8) initially described the IPA in 1904 as "Ciuffo pilorico inferior". Kuroda et al. (9) identified it as the "pyloric branch" in 1982. The term "infrapyloric artery" was first cited in 1988, referring to the IPA originating from the RGEA (10). Recently, investigators have gained further knowledge about the IPA. Sawai et al. (11) used celiac angiograms to study the origin of the IPA. Their results demonstrate that the IPA originates from the GDA (64\%), ASPDA (21\%), and RGEA (12\%) (11). Haruta et al. (12) reported that 156 patients with gastric cancer undergoing laparoscopic surgery had different results of the IPA's origin. This study found that the IPA commonly originated from the ASPDA (64.2\%) and was associated with a specific number of lymph nodes (12). Therefore, the origin of the IPA is still a controversial issue. The current viewpoint is that the IPA feeds the pylorus and the large curvature of the antrum. The location of the origin is the GDA, RGEA, or ASPDA, but the dominant vessel of origin varies across studies.

Previous studies primarily detected the IPA based on

Table 1. Origin of Inferior Pyloric Artery as Detected by Computed Tomography Arteriography and Surgery, Respectively

\begin{tabular}{|c|c|c|c|c|c|c|c|}
\hline \multirow{2}{*}{ CTA Findings } & \multicolumn{7}{|c|}{ Surgical Findings } \\
\hline & Total & ASPDA & GDA & RGEA & ASPDA + RGEA & RGEA + GDA & Not Detected \\
\hline ASPDA & 20 & 20 & & & & & \\
\hline GDA & 4 & & 4 & & & & \\
\hline RGEA & 24 & 1 & 1 & 21 & & & 1 \\
\hline ASPDA + RGEA & 3 & 2 & & 1 & & & \\
\hline RGEA + GDA & 0 & & & & & & \\
\hline Not detected & 3 & 1 & & & & 1 & 1 \\
\hline Total & 54 & 24 & 5 & 22 & 0 & 1 & 2 \\
\hline
\end{tabular}

ASPDA = anterior superior pancreaticoduodenal artery, $C T A=$ computed tomography arteriography, GDA = gastroduodenal artery, RGEA = right gastroepiploic artery 
surgery or celiac arteriography; the surgeons did not perform non-invasive examinations that could reveal the IPA before the operation. CTA is a noninvasive technique for observing the celiac artery and could thus be combined with routine $\mathrm{CT}$ examinations for the preoperative staging of gastric cancer. Previous studies speculated that CTA could detect the main gastric blood supply artery and reveal arterial variations. In addition, CTA can be implemental in gastrectomy. However, currently, there is no study on the application of CTA to observe the IPA.

The current results show that CTA had a good identification rate of IPA, reaching $94.4 \%$ (51/54). We found that the diameters of the IPAs were always small, often $<1 \mathrm{~mm}$. The density of the contrast agent was insufficient after the enhanced scan. Conventional MIP or three-dimensional vascular reconstruction can successfully detect relatively large arteries such as the left gastric artery and the gastroepiploic artery. However, none of these usual methods can detect the IPA, due to its small diameter and the partial volume effect. Our method enabled us to observe the IPA on CT arterial phase images of $0.625-\mathrm{mm}$ thickness. The thin-slice images showed arterial blood vessels with a diameter $<1 \mathrm{~mm}$. Radiologist looked for the artery entering the anterior and posterior wall of the gastric pylorus and defined it as the IPA, and then followed it backwards to search for the original point vessel. At the original point vessel, the MIP image was reconstructed to reveal the anatomical correlations with other adjacent arteries. Finally, we determined the IPA origin by combining the axial thinslice images and the CTA MIP reconstruction images.

In this study, the IPA was not found on CTA in 3 cases; in 1 case, it was not found during surgery, and in 1 case the surgery revealed that the IPA originated from the PDA and the RGEA. The latter patient had undergone distal subtotal

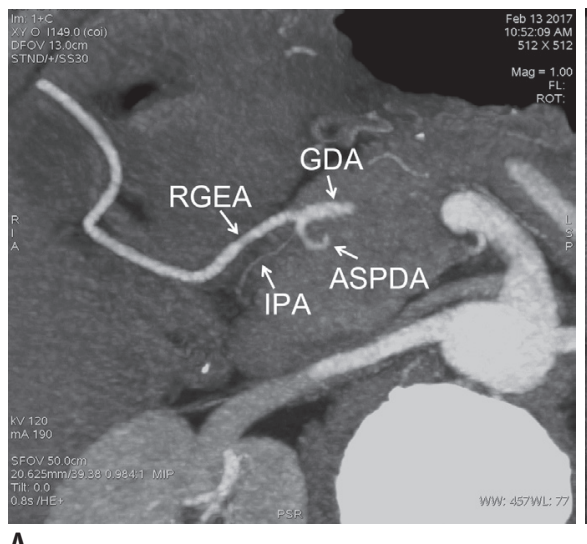

A

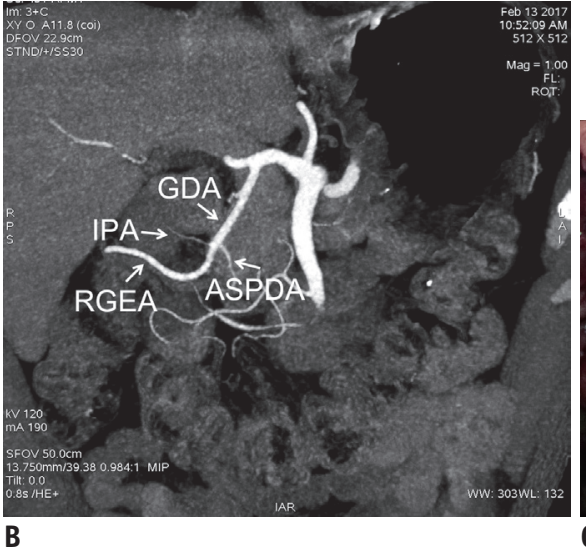

Fig. 1. CTA images show that IPA originated from ASPDA; this was confirmed during surgery.

A, B. MIP construction images show IPA. C. Origin of IPA from ASPDA was confirmed during surgery. ASPDA = anterior superior pancreaticoduodenal artery, CTA = computed tomography arteriography, GDA = gastroduodenal artery, IPA = inferior pyloric artery, $L N=$ lymph node, MIP = maximum intensity projection, RGEA = right gastroepiploic artery

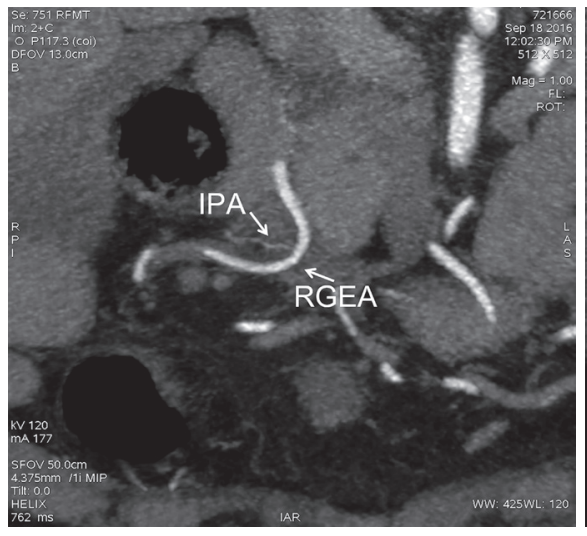

A

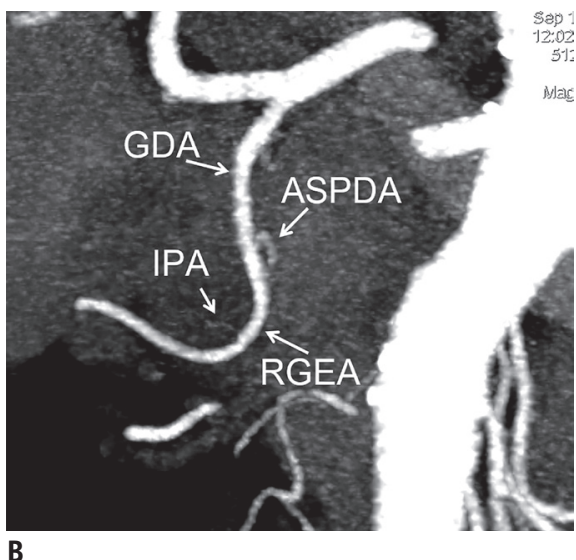

B

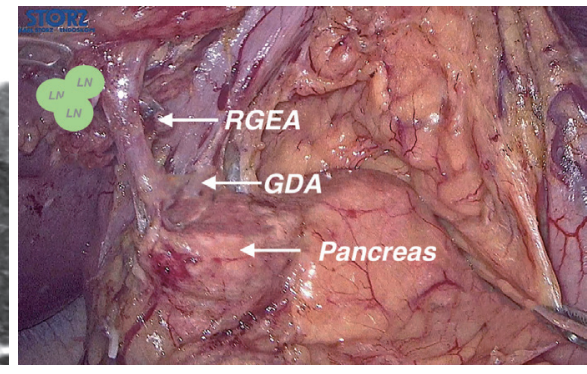

C

Fig. 2. CTA images show that IPA originated from RGEA; however, vessel was not identified during surgery.

A. MIP construction image shows IPA and RGEA. B. RGEA was identified as origin of IPA by CTA. C. Only GDA and RGEA were identified during surgery. 
gastrectomy and Billroth type II anastomosis earlier, because of a benign gastric ulcer. The operation aimed to treat the remnant gastric cancer. As the normal structure of the pylorus had been destroyed during the first operation, CTA did not reveal the exact IPA, while small residual proximal structures of the IPA were identified during the operation. In another case, the patient did not have enough abdominal fat to reveal the large space between the pyloric area and the pancreatic head. Moreover, the hypotonic drugs did not efficiently restrain gastric peristalsis in this patient. The gastric motion artifacts in the pyloric region made it difficult to identify the blood vessels clearly.

During surgery in this case, the surgeons found that the IPA originated from the ASPDA.

The results of the current study show that in 46 cases, the origin of the IPA identified by CTA was in agreement with that revealed during the operation. The diagnostic accuracy of CTA reached $85.2 \%$ (46/54), which suggests that CTA can accurately detect the origin of the IPA preoperatively (Fig.
1). CTA can help the surgeon precisely separate the vessels to preserve the blood supply of the pylorus. The surgical findings of the current study showed that the main origin vessels of the IPA were the ASPDA $(44.4 \%, 24 / 54)$ and the RGEA $(40.7 \%, 22 / 54)$.

In 1 case of a patient whose CTA findings did not match the results of the operation, the IPA was not found during surgery; however, CTA could detect it and revealed that it originated from the RGEA (Fig. 2). We assume that the local tissue structure of the peripyloric area might have been destroyed when the surgeon separated the tissues during the operation and did not find the IPA, while CTA before surgery clearly detected the IPA. This case suggests that CTA can serve as a significant reference for the separation of the IPA during surgery. With preoperative CTA guidance, the surgeons can separate the tiny artery carefully and accurately while avoiding unnecessary damage to the vessels. In 2 cases, the origins of the IPAs were located in the RGEA; however, the origins of the IPAs were found to be
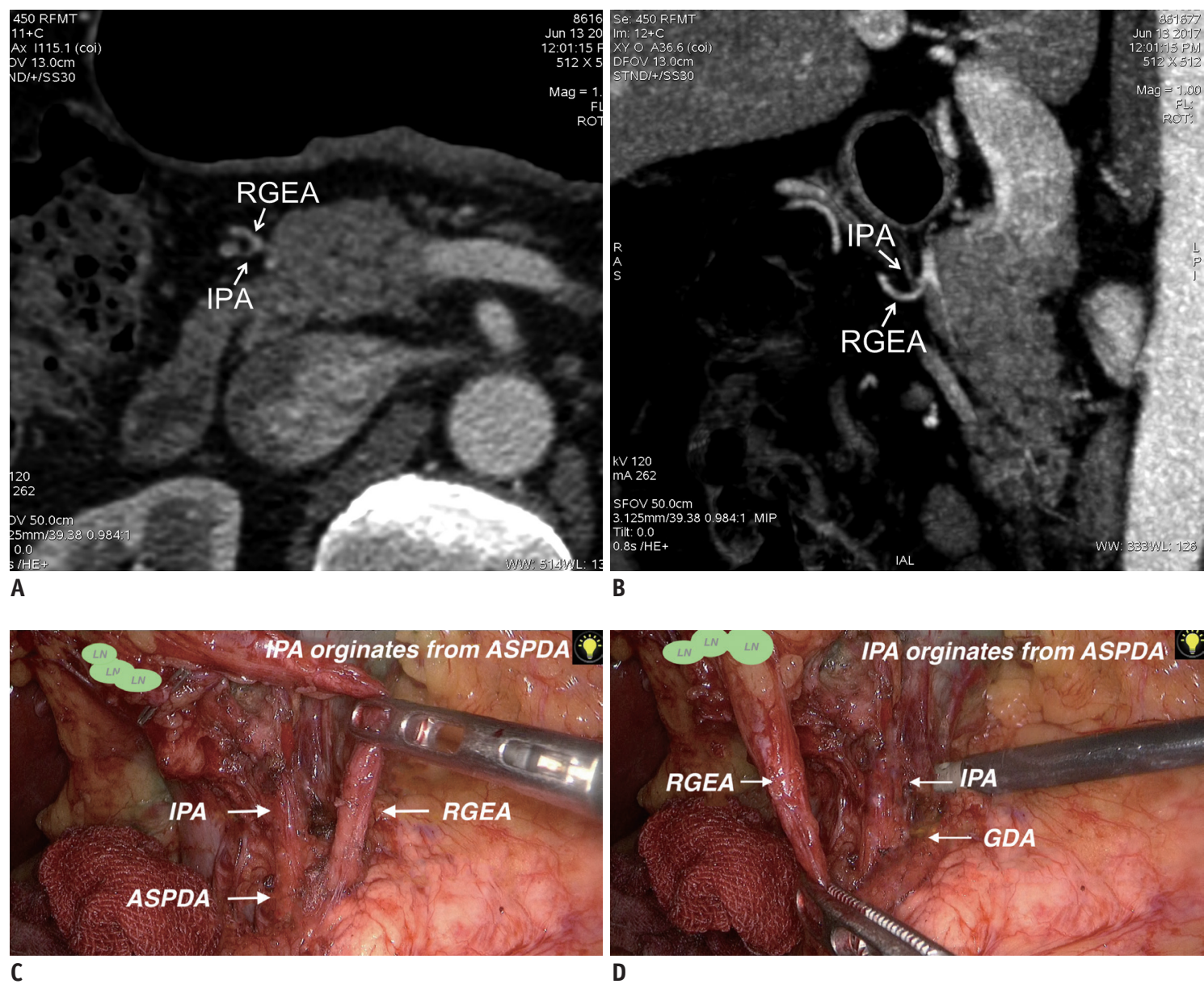

Fig. 3. CTA image of one patient demonstrating origin of IPA to be RGEA; this was not consistent with surgical findings. A. MIP construction images show origin of IPA to be RGEA. B. Oblique sagittal MIP image shows distribution of IPA to pylorus area. C, D. Surgeons judged IPA to have originated from ASPDA. 
the ASPDA and GDA, respectively, during the operation (Fig. 3 ). We conclude that in these cases, the origin point of the IPA might have been proximal to the branch point of the GDA, ASPDA, and RGEA, which made identification by an observer challenging. In 3 cases, the origin of the IPA was determined to be the ASPDA and the RGEA, indicating that the two arteries originated as IPAs. However, the surgical results of these cases showed that the origin of the IPA was the ASPDA or the RGEA alone, as shown in Figure 4 and Table 1. We speculate that one of the origin arteries might have been injured during the operative manipulation, and that only one IPA remained to be found during the surgery. The clinical significance of these cases is that multiple origins of the IPA can be identified by CTA; the surgeon might be prompted to search for all IPAs in order to avoid damage to one of the vessels that affects the blood supply of the pylorus.

We speculate that two factors affected the detection rate of the IPA with CTA. First, the inadequate effect of an injected hypotonic drug might cause gastric motion-related artifacts, which affects the detectability of the IPA. Second, the volume of abdominal fat of the patient can affect the space between the gastric antrum and the pancreas or other adjacent organs. In the case of some lean patients, it was difficult to find the IPA with CTA.

The main limitation of this study is the relatively small sample size, which did not allow to determine the dominant origin vessel of the IPA. The main purpose of the study was to evaluate the feasibility of IPA identification by CTA and to compare the accuracy of this approach with that

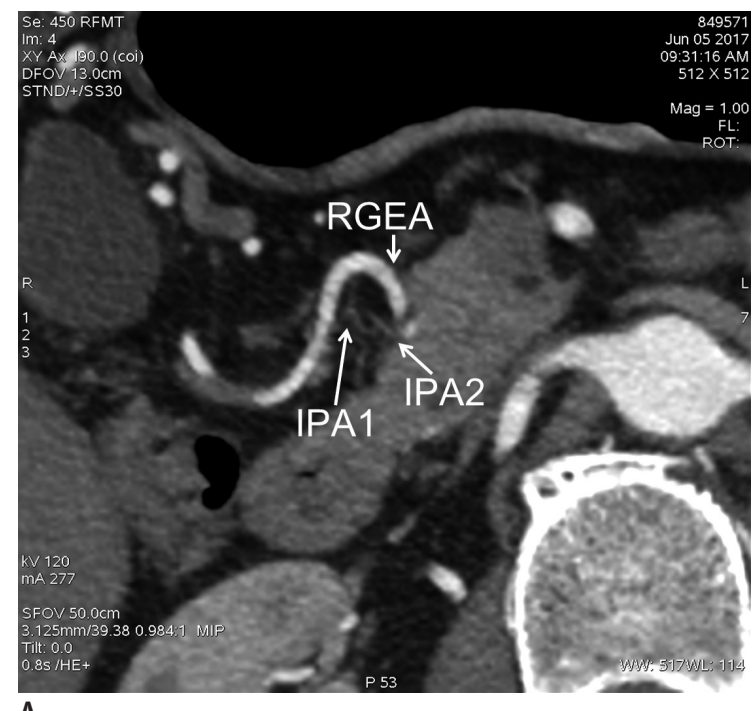

A

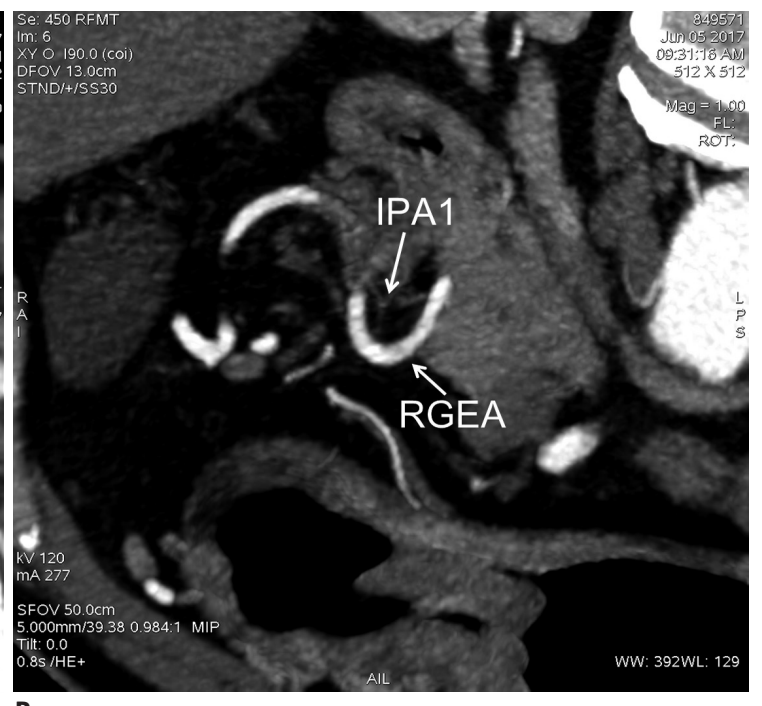

B
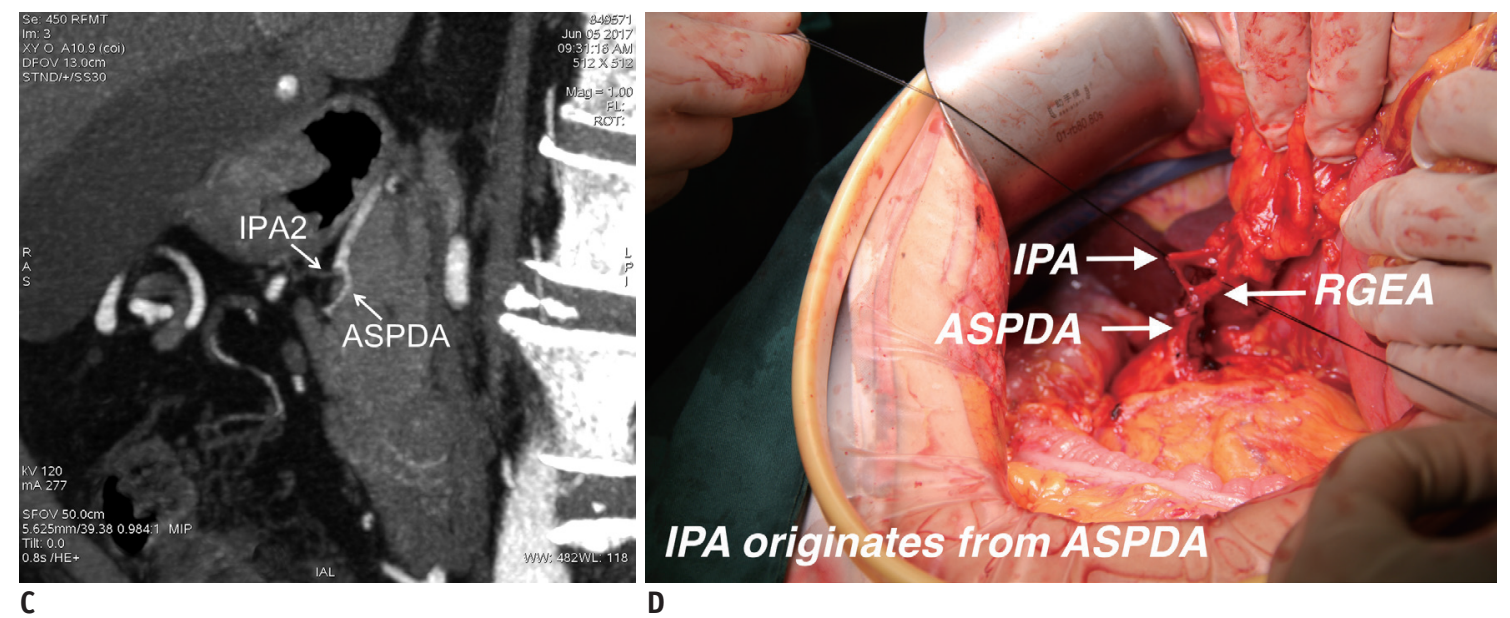

Fig. 4. CTA image of one patient detects two branches of IPA.

A-D. CTA image reveals two branches of IPA, which originate from RGEA and ASPDA, respectively. During surgery, surgeon identified ASPDA as origin vessel for single branch. 
of surgical identification. In order to minimize a potential bias of the surgeon, the identified IPA was not revealed to the surgeon before the surgery. This limited our analysis of whether preoperative identification of the IPA can help surgeons identify the IPA during the operation more accurately. Further trials are still needed to evaluate the practical value of preoperative IPA identification.

In conclusion, we found that CTA can detect the origin of the IPA accurately. The accuracy of CTA, based on the identification of the origin of the IPA during the operation, was $85.2 \%$. Also, CTA identify multiple IPAs, and is thus a critical reference for precise separation and preservation of IPAs during operation. We therefore recommend thinsection CTA as a routine preoperative examination for PPG.

\section{Conflicts of Interest}

The authors have no potential conflicts of interest to disclose.

\section{Acknowledgments}

We thank Kun Cao, Yong Cui, Li-Ping Qi, and Shun-Yu Gao for editorial support and reviewing the manuscript.

\section{ORCID iDs}

Jia-Fu Ji

https://orcid.org/0000-0001-6878-5543

Zhi-Long Wang

https://orcid.org/0000-0002-0268-8246

Ru-Lin Miao

https://orcid.org/0000-0002-7196-1234

\section{REFERENCES}

1. Mizuno A, Shinohara H, Haruta S, Tsunoda S, Kurahashi $Y$, Ohkura $Y$, et al. Lymphadenectomy along the infrapyloric artery may be dispensable when performing pyloruspreserving gastrectomy for early middle-third gastric cancer. Gastric Cancer 2017;20:543-547
2. Shibata C, Saijo F, Kakyo M, Kinouchi M, Tanaka N, Sasaki I, et al.; Society for the Study of Postoperative Morbidity After Gastrectomy. Current status of pylorus-preserving gastrectomy for the treatment of gastric cancer: a questionnaire survey and review of literatures. World J Surg 2012;36:858-863

3. Hiki N, Kaminishi M. Pylorus-preserving gastrectomy in gastric cancer surgery--open and laparoscopic approaches. Langenbecks Arch Surg 2005;390:442-427

4. Iino I, Sakaguchi T, Kikuchi H, Miyazaki S, Fujita T, Hiramatsu $Y$, et al. Usefulness of three-dimensional angiographic analysis of perigastric vessels before laparoscopic gastrectomy. Gastric Cancer 2013;16:355-361

5. Kumano S, Tsuda T, Tanaka H, Hirata M, Kim T, Murakami T, et al. Preoperative evaluation of perigastric vascular anatomy by 3-dimensional computed tomographic angiography using 16-channel multidetector-row computed tomography for laparoscopic gastrectomy in patients with early gastric cancer. J Comput Assist Tomogr 2007;31:93-97

6. Miyaki A, Imamura K, Kobayashi R, Takami M, Matsumoto $J$, Takada Y. Preoperative assessment of perigastric vascular anatomy by multidetector computed tomography angiogram for laparoscopy-assisted gastrectomy. Langenbecks Arch Surg 2012;397:945-950

7. Shinohara H, Kurahashi Y, Kanaya S, Haruta S, Ueno M, Udagawa $\mathrm{H}$, et al. Topographic anatomy and laparoscopic technique for dissection of no. 6 infrapyloric lymph nodes in gastric cancer surgery. Gastric Cancer 2013;16:615-620

8. Rossi G, Cova E. Studio morfolgico delle arterie dello stomaco. Arch Ital Anat Embriol 1904;3:485-657

9. Kuroda C, Nakamura H, Sato T, Yoshioka H, Tokunaga K, Hori S, et al. Normal anatomy of the pyloric branch and its diagnostic significance in angiography. Acta Radiol Diagn (Stockh) 1982;23:479-484

10. Vandamme JP, Bonte J. The blood supply of the stomach. Acta Anat (Basel) 1988;131:89-96

11. Sawai K, Takahashi T, Fujioka T, Minato H, Taniguchi H, Yamaguchi T. Pylorus-preserving gastrectomy with radical lymph node dissection based on anatomical variations of the infrapyloric artery. Am J Surg 1995;170:285-288

12. Haruta S, Shinohara H, Ueno M, Udagawa H, Sakai Y, Uyama I. Anatomical considerations of the infrapyloric artery and its associated lymph nodes during laparoscopic gastric cancer surgery. Gastric Cancer 2015;18:876-880 